\title{
Multiple stacking of InGaAs/GaAs (731) nanostructures
}

\author{
Y. Z. Xie, V. P. Kunets, Z. M. Wang*, V. Dorogan, Y. I. Mazur, J. Wu and G. J. Salamo
}

We studied the multilayering effects of InGaAs quantum dots (QDs) on GaAs(731), a surface lying inside of the stereographic triangle. The surfaces after stacking 16 InGaAs layers were characterized with highly non-uniformity of QD spatial distribution. The bunched step regions driven by strain accumulation are decorated by QDs, therefore GaAs(731) becomes a good candidate substrate for the growth of QD clusters. The unique optical properties of the QD clusters are revealed by photoluminescence measurements. By adjusting the coverage of InGaAs, a bamboo-like nanostructured surface was observed and the quantum dots aligned up in clusters to separate the "bamboo" into sections.

Keywords: Quantum dots; MBE; High index surfaces; Nanostructures; Atomic force microscopy

Citation: Y. Z. Xie, V. P. Kunets, Z. M. Wang, V. Dorogan, Y. I. Mazur, J. Wu and G. J. Salamo, "Multiple stacking of InGaAs/GaAs(731) nanostructures”, Nano-Micro Lett. 1, 1-3 (2009). doi: 10.5101/nml.v1i1.p1-3

The morphological evolution during InGaAs deposition on GaAs high index surfaces has been a subject of numerous experimental efforts for better understanding of growth physics and potential applications of the resulted nanostructures [1-8]. Most of the researches chose a surface index located between two low index planes out of the three, (100), (110), and (111). In other words, the popular choice was a surface index on the sides of the stereographic triangle defined by the three low index planes [3]. For example, $\operatorname{GaAs}(311)$, one of the most popular choice, locates between the (100) and (111) planes. Both quantum wires and quantum dots (QDs) were observed during the InGaAs growth on $\operatorname{GaAs}(311)[1,2,6,8]$. Especially during the processes of stacking multiple layers of InGaAs/GaAs(311) QDs, two-dimensional or one-dimensional arrays of QDs were achieved [8]. In the letter, we study the multilayering effects of InGaAs nanostructures on GaAs(731), a surface index lying within the stereographic triangle. The (731) plane became particularly interesting after the dominating facets of InAs/GaAs(100) QDs were identified to this index [9]. Massive amount of investigations have been performed to reveal the growth behavior of InGaAs on GaAs(100) due to the importance of the resulted QDs for optoelectronic applications [10-12].
Experiments were carried out in a solid source Molecular Beam Epitaxy (MBE) 32P Riber system. The adopted $\operatorname{GaAs}(731)$ substrates were of n-type and epitaxial ready. After oxide desorption, a GaAs buffer layer of $500 \mathrm{~nm}$ was grown at the substrate temperature of $580^{\circ} \mathrm{C}$. The GaAs growth rate was one monolayer per second $(\mathrm{ML} / \mathrm{s})$ under a constant As beam equivalent pressure (BEP) of $1 \times 10^{-5}$ Torr. Sixteen layers of 7 ML $\operatorname{In}_{0.4} \mathrm{Ga}_{0.6}$ As nanostructures were subsequently deposited, partitioned by GaAs spacer in different thicknesses, $120 \mathrm{ML}, 70$ $\mathrm{ML}$, and $50 \mathrm{ML}$, respectively. The growth of $\mathrm{In}_{0.4} \mathrm{Ga}_{0.6}$ As layer was performed at $540^{\circ} \mathrm{C}$ and As BEP of $4.4 \times 10^{-6}$ Torr. After the growth of first 5 MLs of GaAs spacer, the growth temperature was rapidly increased up to $580^{\circ} \mathrm{C}$ and As BEP was turned back to $1 \times 10^{-5}$ Torr. One more sample, grown in addition to the above series, had the GaAs spacer of $70 \mathrm{ML}$ but the $\operatorname{In}_{0.4} \mathrm{Ga}_{0.6} \mathrm{As}$ coverage of 5.7 ML. Following the growths, samples were quenched to room temperature and then taken out of the MBE chambers for Atomic Force Microscopy (AFM) studies under ambient atmosphere. For optical properties of the samples, the photoluminescence (PL) measurements were performed at $10 \mathrm{~K}$ in a closed-cycle helium cryostat. The $532 \mathrm{~nm}$ line from a Nd:YAG (yttrium aluminum garnet) laser was used for continuous-wave (CW) PL excitation. The laser spot diameter 

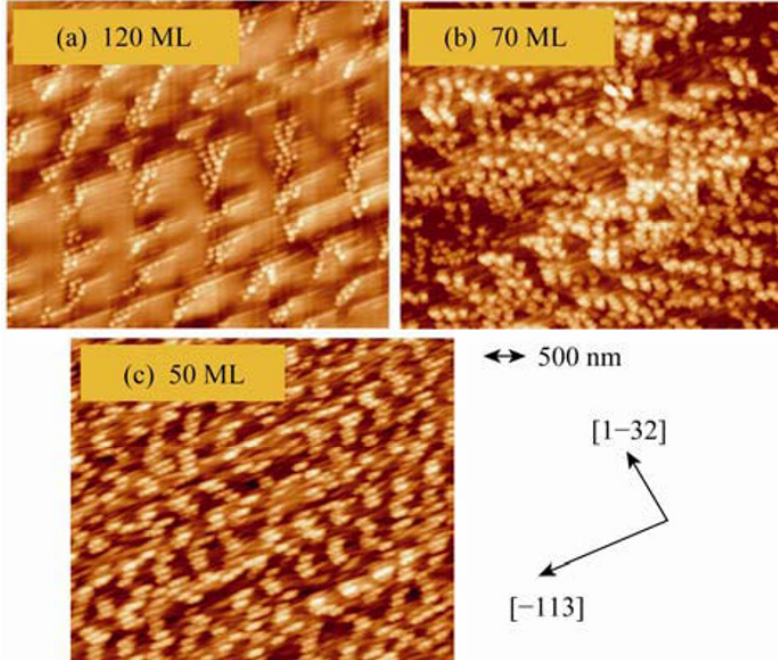

FIG. 1. $5 \times 5 \mu \mathrm{m}$ AFM images of InGaAs/GaAs(731) multilayer with GaAs spacer of $120 \mathrm{ML}, 70 \mathrm{ML}$, and $50 \mathrm{ML}$, respectively. The scale bar of $500 \mathrm{~nm}$ and the lateral direction of [-113] and [1-32] apply to all three images.

was of $\sim 20 \mathrm{~m}$ and the optical excitation power was in the range of $10^{-6} \sim 10^{2} \mathrm{~mW}$. The PL signal from the sample was dispersed by a $0.5 \mathrm{~m}$ single-grating monochromator and detected by a liquid nitrogen (LN)-cooled InGaAs photodiode detector array.

Figure 1(a) shows an AFM image of the surface nanostructures after stacking 16 layers of $7 \mathrm{ML} \operatorname{In}_{0.4} \mathrm{Ga}_{0.6} \mathrm{As}$ spaced by $120 \mathrm{ML}$ GaAs interlayer. The surface is characterized as QD decorated steps and large terraces free of QDs. While (731) was observed as the dominating facets bounding InAs/GaAs(100) QDs, the GaAs(731) surface itself is unstable. $\operatorname{GaAs}(1152)$, just $2.28^{\circ}$ away from $\operatorname{GaAs}(731)$, has lower surface energy according to theoretical calculation and was observed to be a stable surface by scanning tunneling microscopy (STM) [13]. Therefore, $\operatorname{GaAs}(731)$ is better described as a vicinal GaAs(11 5 2) surface, with straight steps along [-113] and ragged steps along [1-32]. The large terraces that are free of QDs shown in Fig. 1(a) resulted from the strain-driven step bunching. It is well documented that QDs prefer to stepped regions for nucleation and growth, especially ragged steps due to its active reaction. The observation in Fig. 1(a) demonstrates that $\operatorname{GaAs}(731)$ is a substrate candidate for the growth of InGaAs QD clusters. The corresponding PL spectrum from this sample is shown in Fig. 2. The strong and sharp peak about $1.38 \mathrm{eV}$ can be assigned to the InGaAs wetting layer (WL) covering the whole surface. The assignment is consistent with the high peak energy and large terraces without QDs. Different from usual QDs reported where the PL peak from $\mathrm{WL}$ is weak, the carriers excited in InGaAs/GaAs(731) WL cannot find nearby QDs to relax and therefore recombine in $\mathrm{WL}$ giving rise to a strong signal. The relatively weak peak around $1.25 \mathrm{eV}$ is expected from the clustered QDs.

By reducing the GaAs spacer thickness from $120 \mathrm{ML}$ to 70 ML, more strain could transmit from the underneath InGaAs layer to the subsequent layers. As a result of the strain-driven mechanism, Fig. 1(b) reveals that the InGaAs QDs grow bigger in size and the free-of-QDs terraces disappear. Consistently, the WL peak disappears from its associated PL spectrum in Fig. 2 and the QD peak around $1.25 \mathrm{eV}$ becomes dominant. Please notice, this peak is from 15 buried layers of QDs. The luminescence from the exposed layer of surface QDs is usually not notable, as in the PL spectrum form the sample with 120 ML spacer. However, the PL contribution from surface QDs can be enhanced as the spacer becomes thinner, as the consequence of carrier tunneling from the buried QDs to the surface. In line with the literature [6], the new developed PL peak around 1.0 $\mathrm{eV}$ is assigned to the layer of surface QDs.

With further reducing the GaAs spacer to $50 \mathrm{ML}$, more strain is accumulated to the surface and the QDs further ripe and even elongate along [-113], as revealed in Fig. 1(c). Due to the thinner spacer, more carriers are able to tunnel from buried layers to the surface. The PL peak from surface QDs becomes stronger, and eventually dominates the spectrum as shown in Fig. 2.

As noticed, the amount of accumulated strain is the key in determining the nanostructure configuration in Fig. 1 and the optical properties in Fig. 2. Specially, the sample with less accumulated strain shown in Fig. 1(a) demonstrated the potential of $\operatorname{GaAs}(731)$ for growing QD clusters. Therefore, to pursue better QD clusters, we introduce a new sample with less InGaAs coverage, 5.7 ML, in order to significantly reduce the

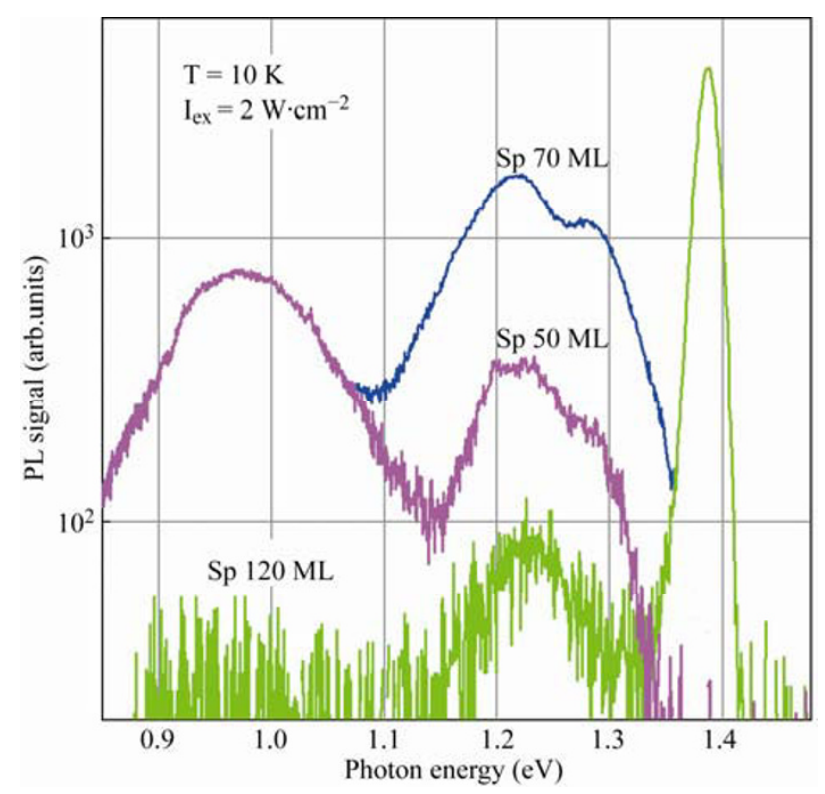

FIG. 2. PL spectra of InGaAs multilayers spaced by GaAs in different thickness. 


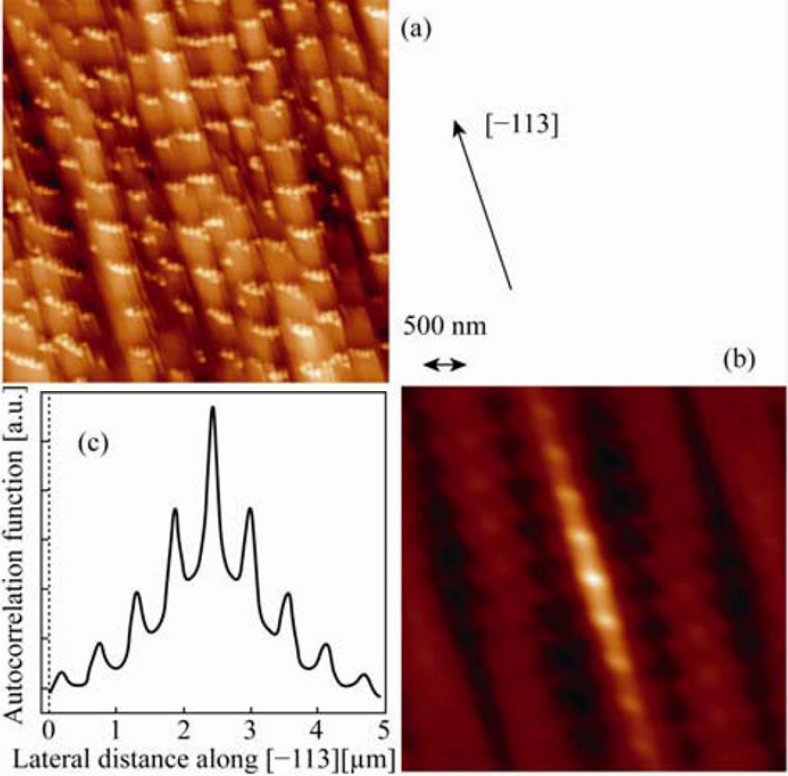

FIG. 3. (a) $5 \times 5 \mu \mathrm{m}$ AFM image of InGaAs/GaAs(731) multilayers with $\mathrm{InGaAs}$ coverage of 5.7 ML and GaAs spacer of 70 ML. (b) Two-dimensional image of autocorrelation functions calculated from (a). (c) A line profile along [-113] crossing the center of (b). The scale bar of $500 \mathrm{~nm}$ and the lateral direction of [-113] apply to both (a) and (b).

total strain. The result is shown in Fig. 3. Comparing Fig. 3(a) to Fig. 1(b), two samples with the same spacer thickness of 70 ML, we are able to rebuild the QD clusters by reducing the accumulated strain. The surface is characterized by steps plus terraces again and QD clusters decorate the ragged steps. Remarkably, the QDs in clusters are aligned as short chains. The visual appearance of the resulted surface morphology resembles itself as a jungle of bamboo. The bunched steps along [-113] visualize the individual bamboo sticks and aligned QD clusters separate each stick into sections. Very interesting, the QD clusters are nearly equally distributed along [-113]. Its periodicity is better resolved in the two-dimensional image of the autocorrelation functions calculated from the AFM image [14]. Figure 3(c) shows a line profile along [-113] through the center of Fig. 3(b). The separation between satellite peaks reveals that the periodicity is about $565 \mathrm{~nm}$. The separations between "bamboos" are not uniform but still resolved from Fig. 3(b), around $1.08 \mu \mathrm{m}$.

In conclusion, we stacked multilayer of InGaAs nanostructures on $\operatorname{GaAs}(731)$, and studied the effect by varying GaAs spacer thickness and InGaAs coverage. The resulted surface morphologies and optical properties are sensitive to the accumulated strain. $\operatorname{GaAs}(731)$ is demonstrated as a good candidate for growing QD clusters. The possibility of QD ordering inside clusters is revealed by AFM for low coverage of InGaAs. Our report indicates the need to study InGaAs growth on GaAs surfaces lying inside the stereographic triangle, thus achieve a comprehensive picture of strain-driven growth and pursuing novel QD configurations.

Received 16 August 2009; accepted 31 August 2009; published online 15 October 2009.

\section{References}

1. S. Sanguinetti, S. Castiglioni, E. Grilli, M. Guzzi, G. Panzarini, L. C. Andreani and M. Henini, Jpn. J. Appl. Phys. 38, 4676 (1999). doi:10.1143/JJAP.38.4676

2. H. Wen, Z. M. Wang and G. J. Salamo, Appl. Phys. Lett. 84, 1756 (2004). doi:10.1063/1.1664018

3. Y. Temko, T. Suzuki, M. C. Xu and K. Jacobi, Surf. Sci. 591, 117 (2005). doi:10.1016/j.susc.2005.06.026

4. Y. Temko, T. Suzuki, M. C. Xu, K. potschke, D. Bimberg and K. Jacobi, Phys. Rev. B 71, 045336 (2005).

5. M. Zervos, C. Xenogianni, G. Deligeorgis, M. Androulidaki, P. G. Savvidis, Z. Hatzopoulos and N. T. Pelekanos, Phys. Status Solidi C 3, 3988 (2006).

6. B. L. Liang, Zh. M. Wang, Yu. I. Mazur, Sh. Seydmohamadi, M. E. Ware and G. J. Salamo, Optics Express 15, 8157 (2007). doi:10.1364/OE.15.008157

7. C. Wang, Y. Yang, X.M. Chen, Z.L. Liu, H.Y. Cui, S. Zhang, X.S. Chen and W. Lu, Appl. Surf. Sci. 254, 4626 (2008). doi:10.1016/j.apsusc.2008.01.089

8. Zh. M. Wang, C. Rodriguez, Sh. Seydmohamadi, Yu. I. Mazur, Y. Z. Xie and G. J. Salamo, Appl. Phys. Lett. 94, 083107 (2009). doi:10.1063/1.3089695

9. J. Marquez, L. Geelhaar and K. Jacobi, Appl. Phys. Lett. 78, 2309 (2001). doi:10.1063/1.1365101

10. D. Leonard, M. Krishnamurthy, C. M. Reaves, S. P. Denbaars and P. M. Petroff, Appl. Phys. Lett. 63, 3203 (1993). doi:10.1063/1.110199

11. D. Bimberg, M. Grundmann and N. N. Ledentsov, Quantum Dot Heterostructures (Wiley, Chichester, 1999).

12. S. S. Li, J. B. Xia, J. L. Liu, F. H. Yang, Z. C. Niu, S.L. Feng and H.Z. Zheng, J. Appl. Phys. 90, 6151 (2001). doi: $10.1063 / 1.1416855$

13. L. Geelhaar, J. Marquez, P. Kratzer and K. Jacobi, Phys. Rev. Lett. 86, 3815 (2001). doi:10.1103/PhysRevLett.86. $\underline{3815}$

14. Z. M. Wang, J. L. Shultz and G. J. Salamo, Appl. Phys. Lett. 83, 1749 (2003). doi:10.1063/1.1606891 